\title{
One Dimensional Local Binary Patterns of Electroencephalogram Signals for Detecting Alzheimer's Disease
}

\author{
Santosh Tirunagari*, Samaneh Kouchaki ${ }^{\ddagger}$, Daniel Abasolo ${ }^{\dagger}$, and Norman Poh* $^{*}$ \\ * Department of Computer Science. \\ $\dagger$ Department of Mechanical Engineering Sciences. \\ ${ }^{*}$ Faculty of Engineering and Physical Sciences, University of Surrey, Guildford, Surrey, United Kingdom. \\ $\ddagger$ Computational Health Informatics, Department of Engineering Science, University of Oxford, United Kingdom.
}

\{santosh.tirunagari, d.abasolo, n.poh\}@ surrey.ac.uk.

\begin{abstract}
Alzheimer's disease (AD) is neurodegenerative, caused by the progressive death of brain cells over time. One non-invasive approach to investigate $\mathrm{AD}$ is to use electroencephalogram (EEG) signals. The data are usually non-stationary with a strong background activity and noise which makes the analysis difficult leading to low performance in many real world applications including the detection of AD. In this study, we present a method based on local texture changes of EEG signals to differentiate $\mathrm{AD}$ patients from the healthy ones, using one-dimensional local binary patterns (1D-LBPs) coupled with support vector machines (SVM). Our proposed method maps the EEG data into a less detailed representation which is less sensitive to noise. A 10 fold cross validation performed at both the epoch and subject level show the discriminancy power of 1D-LBP feature vectors with application to AD data.
\end{abstract}

\section{INTRODUCTION}

Alzheimer's disease (AD) is the most frequent cause of dementia in the western world, and is caused by the progressive death of brain cells over time (neurodegeneration) affecting an individual's cognitive ability as well as influencing their psychological capacity [1]. Clinical diagnoses of AD are made primarily on the basis of medical history, psychiatric evaluation and memory and mental health tests [2], although an indisputable diagnosis is only possible through necropsy [3]. While symptoms and their severity vary from patient to patient, early diagnosis is vital for reducing their effects with available pharmacological treatments and through life style adjustments [4].

Electroencephalograms (EEGs) have been used in the investigation of dementia for several decades [5]-[8]. As EEG signals reflect brain electrical activity, they can be used to detect electrical abnormalities in brain signals with non-invasive cranial surface electrodes [9]. Generally EEGs in AD patients show a shift to lower frequencies in spectral analysis, which suggests a decreased cohesion of cognitive networks [10]. Moreover, AD patients' EEGs display less complexity and contain more regular patterns compared to those of control subjects [9], [11]-[13]. Due to the intrinsic irregular and aperiodic nature of EEG signals, spectral analysis techniques may be insufficient for characterising the dynamics of the events underlying the EEG signals. Thus, additional techniques, have been developed, including non-linear time series analysis [14] (particularly utilising entropy based methods [11]-[13]), for automatically distinguishing $\mathrm{AD}$ patients from control ones.
Recently in [15] such methods were utilised as features for a machine learning classifier used to automatically distinguish $\mathrm{AD}$ patients from control ones.

In this study, we present a feature extraction algorithm based on one-dimensional local binary patterns (1D-LBPs) for extracting quantitative histograms from EEG signals. LBPs have been extensively used for texture analysis of images [16], [17], which are 2D in nature. LBP has become a popular method due to its fast processing requiring substantially less computational power and its strong discriminative capabilities. The use of 1D-LBP for signal processing was introduced by Chatlani et al. [18] to detect voice activity in nonstationary speech signals. Since EEG signals are also nonstationary in nature, we applied 1D-LBPs in order to extract patient specific signatures that are unique and salient features when compared to controls. Kaya et al. [19] introduced the approach of using 1D-LBP for feature extraction of raw EEG signals coupled with a machine learning classifier. Their results demonstrated a high classification accuracy of epileptic EEG signals. Texture representation of timefrequency (tf) imagebased epileptic seizure detection was also proposed [20], [21] using 2D-LBP. Here, we show LBP provides an alternative feature space to classify AD from healthy subjects. Moreover, the effect of LBP parameter selection, electrode selection, and epoch and subject level evaluation on the performance are examined.

Contributions of the paper are as follows: Although LBP has been used extensively as a feature descriptor in the fields of image [16], [17], [22], [23], speech [18], [24] and signal processing [18], [25], its application to analysing AD patients' data is novel. We show that $1 \mathrm{D}-\mathrm{LBP}$ captures the descriptive information, in terms of histograms representing the relative changes in EEG amplitudes, in a way that can be readily utilised by a support vector machine (SVM) for classification.

For evaluating the classification performance, we introduce the half total error rate (HTER) for the first time in the field of EEG signal processing. Although HTER has been used extensively in the field of biometrics [22], [23], its usage in biomedical applications is not very well known. HTER has the advantage of not being affected by the overwhelmingly large sample size of one class versus another because both types of errors are weighted equally, thus coercing equal contributions from both errors. Therefore, it has an advantage 
over traditional performance evaluation techniques such as the F-score or accuracy.

The organisation of the paper is as follows: In section II we present the methodology and in Section III the dataset is described. In section II we present the 1D-LBP methodology as well as the HTER evaluation criteria. Experiments and results are discussed in section IV, including data preprocessing. Finally, in section $\mathrm{V}$ we discuss the conclusions that can be drawn from the results.

\section{Methodology}

In this section we present 1D-LBP as applied to EEGs, and use SVMs with an RBF kernel to distinguish between healthy and $\mathrm{AD}$ patients. The classification performance is then evaluated by the minimum HTER criteria.

\section{A. 1D-Local Binary Patterns}

LBP, introduced by Ojala et al. [17], is a powerful operator that extracts micro-texture information invariant to local grey scale variations, and has gained significant popularity in the field of image, speech and signal processing [16]. Using LBP, each two-dimensional segment (window) is mapped to a binary string with a fixed length. An LBP code summarises the textural changes in images and frequency changes in speech, while its histogram distribution shows how often each pattern appears. These histograms are taken to be the feature vector that represents the EEG signatures for the pathology or control.

LBP assigns a binary code to each sample by examining its neighbouring points. By considering $x(t)$ as the $t^{t h}$ sample of the numerical representation of an EEG signal, LBP can be defined as:

$$
\begin{array}{r}
\operatorname{LBP}(x(t))=\sum_{i=0}^{p / 2-1}\left\{\operatorname{Sign}(x(t+i-p / 2)-x(t)) 2^{i}+\right. \\
\left.\operatorname{Sign}(x(t+i+1)-x(t)) 2^{i+p / 2}\right\},
\end{array}
$$

where $p$ is the number of neighbouring points and Sign indicates the sign function:

$$
\operatorname{Sign}(x)=\left\{\begin{array}{ll}
0 & x<0 \\
1 & x \geq 0
\end{array} .\right.
$$

Sign is used to assign a binary number by thresholding the difference between each neighbouring point and the centre point $t$. Consequently, each segment (window) of length $p+1$ is assigned a $p$-bit binary number. Each binary number is converted to a LBP code using a binomial weight. An example of the LBP operator can be seen in Figure 1 where $p=6$. The value of the centre point (in the square in Figure 1) is compared with the six neighbouring points to produce the LBP code. This code describes the data changes locally in a compressed format. Finally, by considering all the obtained codes, the distribution of the LBP codes can be defined as

$$
\mathbf{h}_{k}=\sum_{p / 2 \leq i \leq N-p / 2} \delta\left(\operatorname{LBP}_{p}(x(i), k),\right.
$$

where $k=1,2, \ldots, 2^{p}$ and $N$ is the signal length. Considering the distribution makes the feature space independent on the location of each pattern. Here, we apply LBP to one-dimensional non-stationary EEG signals to extract pattern changes.

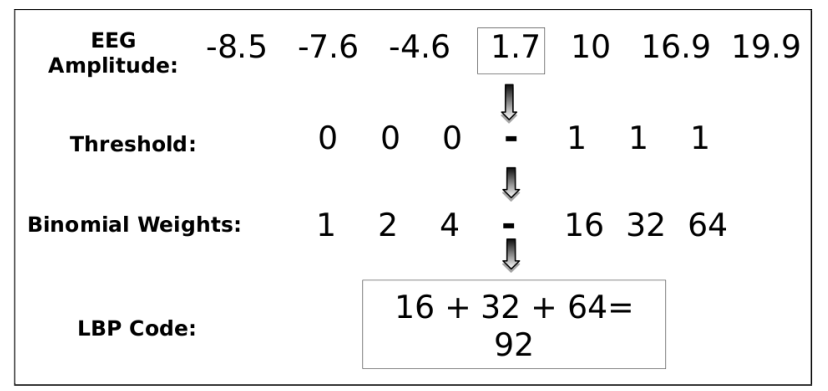

Fig. 1. Calculating the LBP code. A thresholding of the EEG amplitude of a size 7 segment is determined by comparing the centre point (in the square) and its neighbours. The LBP code is then obtained using binomial weights.

\section{B. Classification Details}

The LBP histograms are given as an input to the SVMs, as they are known for their high classification accuracy. For the SVMs, the radial basis function (RBF) kernel was used with kernel scale $\sigma=1$ and box constraint value set to 1 . The performance was evaluated using 20 repeated 10-fold cross validation.

\section{Performance and Threshold Criteria}

Although the output of an SVM can be used to make a hard decision, we use a soft output, defined as the distance of a test sample from the SVM decision hyperplane. For all experiments, we label samples derived from AD patients' EEG signals as positive, and those derived from the healthy subjects (i.e., the control) as negative.

Let $\mathcal{T}$ be the domain of the SVM output. A decision is made by comparing the SVM output $t \in \mathcal{T}$ with a decision threshold, $\Delta \in \mathcal{T}$, as follows:

$$
\operatorname{decision}(t)=\left\{\begin{array}{cl}
\text { positive } & \text { if } t>\Delta \\
\text { negative } & \text { otherwise }
\end{array}\right.
$$

Let $\mathcal{T}_{+} \subset \mathcal{T}$ be the set of scores for the positive samples; and $\mathcal{T}_{-} \subset \mathcal{T}$, the negative samples. By applying the decision threshold $\Delta$, the system can commit two types of errors, namely, false rejection rate (FRR) and false acceptance rate (FAR). Their respective error rates are calculated as follow:

$$
\begin{aligned}
\operatorname{FRR}(\Delta) & \equiv P\left(t \leq \Delta \mid \mathcal{T}_{+}\right) \\
& \approx \frac{\left|\left\{t \in \mathcal{T}_{+}, t \leq \Delta\right\}\right|}{\left|\mathcal{T}_{+}\right|} \\
\operatorname{FAR}(\Delta) & \equiv 1-P\left(t \leq \Delta \mid \mathcal{T}_{-}\right), \\
& \approx \frac{\left|\left\{t \in \mathcal{T}_{-}, t>\Delta\right\}\right|}{\left|\mathcal{T}_{-}\right|}
\end{aligned}
$$

Note that the FRR is an increasing function of the decision threshold, whereas FAR is a decreasing function of it.

By plotting FRR versus FAR curve, one obtains a receiver's operating characteristic (ROC) curve. There are several useful point-estimate criteria that are useful to find the operational decision threshold, namely, equal error rate (EER), weighted error rate (WER) and its special case, half-total error rate (HTER). 
The first one, EER, is the point where the two error rates cross each other:

$$
\operatorname{EER}\left(\Delta_{*}\right)=\operatorname{FAR}\left(\Delta_{*}\right)=\operatorname{FRR}\left(\Delta_{*}\right)
$$

where $\Delta_{*} \in \mathcal{T}$ is the unique decision threshold of the EER. In practice, the EER is found by searching for a threshold that minimises the absolute difference between the FAR and FRR.

$$
\Delta_{E E R}=\arg \min _{\Delta}|\operatorname{FAR}(\Delta)-\operatorname{FRR}(\Delta)|
$$

The second threshold-optimizing criterion is WER, and is defined as:

$$
W E R(\Delta)=\alpha \times \operatorname{FAR}(\Delta)+(1-\alpha) \times \operatorname{FRR}(\Delta)
$$

When $\alpha=\frac{1}{2}$, we have the HTER.

Throughout the experiments, we shall use EER to optimize the decision threshold and report performance in HTER [22], [23].

The significant advantage of reporing the performance in HTER, is that it is not affected by the imbalance in the number of samples in each class as both types of errors are weighted equally, thus ensuring equal contribution from both errors (i.e. enforcing equal prior probability for both classes). In our case, this is particularly desirable as the prior probability of a patient's EEG signal is difficult to estimate in practice. In the absence of any additional information, using equal prior probabilities is a reasonable option.

\section{DATASET}

The database used in this pilot study included 11 patients with a diagnosis of probable $\mathrm{AD}$ ( 5 men; 6 women; age: $72.5 \pm 8.3$ years, mean \pm standard deviation (SD)) and 11 agematched controls ( 7 men; 4 women; age: $72.8 \pm 6.1$ years, mean $\pm \mathrm{SD}$ ). The average Mini-Mental State Examination (MMSE) score for the AD patients was $13.1 \pm 5.9$ (mean $\pm \mathrm{SD}$ ). All control subjects had an MMSE score of 30 .

AD patients were recruited from the Alzheimer's Patients' Relatives Association of Valladolid (AFAVA), Spain. Informed consent was obtained for the recording of the EEG signals at the Hospital Clnico Universitario de Valladolid (Spain) using the international $10-20$ system and electrodes (Fp1, Fp2, F3, F4, C3, C4, P3, P4, O1, O2, F7, F8, T3, T4, T5, T6, $\mathrm{Fz}, \mathrm{Cz}$ and $\mathrm{Pz}$ ) referenced to the linked ear lobes and with subjects sitting on a chair and with eyes closed. More than 5 minutes of EEG data were recorded for each subject using Oxford Instruments Profile Study Room 2.3.411 (Oxford, UK). A hardware low-pass filter with a cut-off frequency of $100 \mathrm{~Hz}$ was used before signals were sampled at $256 \mathrm{~Hz}$ and digitised with a 12-bit A-to-D converter. Five second epochs with little artefactual activity were selected for offline analysis by a specialist neurophysiologist. The total number of artefact-free epochs available for analysis was 9849 (5648 from AD patients and 4201 from control subjects). On average, $28.0 \pm 15.1$ epochs (meanSD) were available from each electrode and each subject.

\section{EXPERIMENTS AND RESULTS}

In this section we present our experiments and results. Our preliminary observation from the $1 \mathrm{D}-\mathrm{LBP}$ histograms of $\mathrm{AD}$ patients and controls have been considered. It shows that the intensity levels differ between the groups (Figure 2), with AD patients' 1D-LBP histograms having greater levels of intensity when compared to the controls. In this study, we therefore propose to apply 1D-LBP to EEGs and use SVMs with an RBF kernel to distinguish between controls and AD patients.
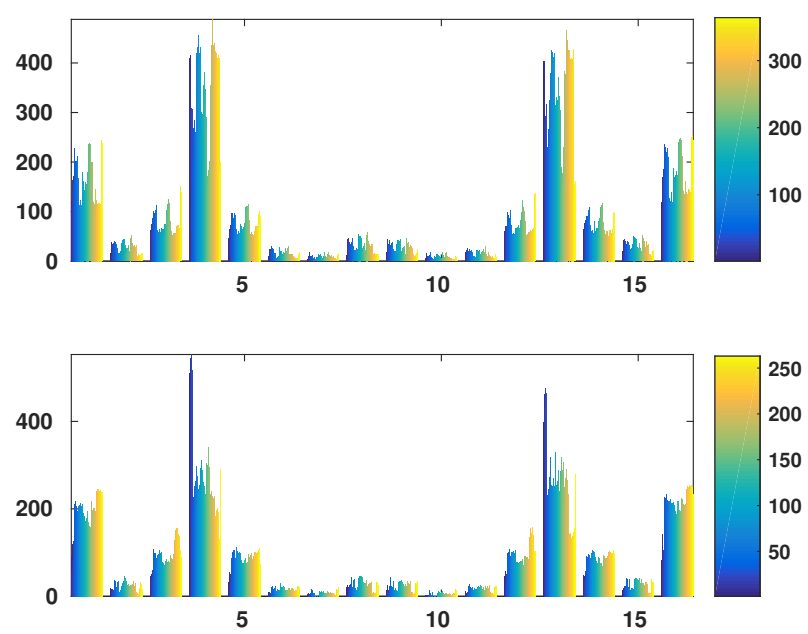

Fig. 2. LBP histogram codes for electrode $\mathbf{O 2}$ in Alzheimer's patients (top) and healthy subjects (bottom). The AD patients have 363 epochs while healthy ones include 264 epochs.

Moreover, the effect of following experiments have been considered on the performance of the proposed method:

1) The choice of LBP parameters. In order to use LBP, we first have to determine the optimal segment length to use. Therefore, in this experiment we investigate the optimal segment length for maximising performance in terms of HTER.

2) Importance of electrodes. In this experiment we investigate the performance obtained using each electrode, in terms of the HTER.

3) Cross subject evaluation. Instead of performing cross-validation at the epoch level, where the training and test set may contain EEG signals from the same subjects, in this experiment by using subject-level cross-validation, we ensure that the training and test sets (on a different fold) are two distinctive partitions with data from different subjects.

For both experiments 2 and 3 above the optimal parameters identified in experiment 1 were used. The results of these experiments are reported in the subsections below.

\section{A. Impact of $L B P$ parameters on the classification}

In order to find the best parameters for the 1D-LBP, we ran 20 repetitions of a 10 -fold cross validation across all 16 electrodes. The results in Table I present the means and standard deviations of the HTER across the 20 runs of 10 fold cross validation. From Table I we see that 1D-LBP with a segment size of 5 gave the best performance (i.e., with the minimum HTER value) when compared to other segment sizes. 
TABLE I. MEANS AND STANDARD DEVIATIONS OF THE HTER (FOR EACH ELECTRODE) ACROSS 20 RUNS OF 10 FOLD CROSS VALIDATION.

\begin{tabular}{|c|c|c|c|c|}
\hline Electrode & \multicolumn{4}{|c|}{ LBP segment size ( size of feature vector) } \\
\hline & $\mathbf{3} \mathbf{( 4 )}$ & $\mathbf{5}(\mathbf{1 6})$ & $\mathbf{7}(\mathbf{6 4})$ & $\mathbf{9}(\mathbf{2 5 6})$ \\
\hline C3 & $0.22 \pm 0.05$ & $0.14 \pm 0.05$ & $0.20 \pm 0.05$ & $0.21 \pm 0.07$ \\
\hline C4 & $0.25 \pm 0.04$ & $0.14 \pm 0.05$ & $0.19 \pm 0.04$ & $0.20 \pm 0.10$ \\
\hline F3 & $0.28 \pm 0.05$ & $0.20 \pm 0.07$ & $0.26 \pm 0.05$ & $0.23 \pm 0.10$ \\
\hline F4 & $0.32 \pm 0.05$ & $0.24 \pm 0.08$ & $0.29 \pm 0.05$ & $0.34 \pm 0.11$ \\
\hline F7 & $0.26 \pm 0.05$ & $0.18 \pm 0.05$ & $0.27 \pm 0.05$ & $0.36 \pm 0.13$ \\
\hline F8 & $0.25 \pm 0.05$ & $0.17 \pm 0.05$ & $0.24 \pm 0.05$ & $0.31 \pm 0.12$ \\
\hline Fp1 & $0.25 \pm 0.05$ & $0.18 \pm 0.05$ & $0.27 \pm 0.05$ & $0.28 \pm 0.13$ \\
\hline Fp2 & $0.26 \pm 0.05$ & $0.14 \pm 0.04$ & $0.23 \pm 0.05$ & $0.22 \pm 0.14$ \\
\hline O1 & $0.28 \pm 0.05$ & $0.19 \pm 0.05$ & $0.25 \pm 0.05$ & $0.23 \pm 0.13$ \\
\hline O2 & $0.29 \pm 0.05$ & $\mathbf{0 . 1 2} \pm \mathbf{0 . 0 4}$ & $0.20 \pm 0.05$ & $0.23 \pm 0.15$ \\
\hline P3 & $0.27 \pm 0.05$ & $0.14 \pm 0.04$ & $0.21 \pm 0.04$ & $0.28 \pm 0.10$ \\
\hline P4 & $0.22 \pm 0.05$ & $0.13 \pm 0.05$ & $0.22 \pm 0.05$ & $0.26 \pm 0.10$ \\
\hline T3 & $0.26 \pm 0.05$ & $0.20 \pm 0.06$ & $0.25 \pm 0.05$ & $0.35 \pm 0.14$ \\
\hline T4 & $0.27 \pm 0.05$ & $0.20 \pm 0.05$ & $0.25 \pm 0.05$ & $0.29 \pm 0.20$ \\
\hline T5 & $0.28 \pm 0.05$ & $0.19 \pm 0.06$ & $0.29 \pm 0.05$ & $0.28 \pm 0.15$ \\
\hline T6 & $0.29 \pm 0.05$ & $0.23 \pm 0.06$ & $0.27 \pm 0.05$ & $0.33 \pm 0.13$ \\
\hline
\end{tabular}

\section{B. Importance of Electrodes}

The electrode $\mathbf{C 4}$ gave a minimum HTER of 0.08 across the 20 runs of 10 fold cross validation, followed by electrode Fp2. Although the results on the aforementioned electrodes were minimal, they lie outside the interquartile range of the median analysis, thus suggesting their values to be outliers. Electrode O2 gave a consistent result with a HTER between the range of 0.08 and 0.16 across the 20 runs (Figure 3 ). Therefore, from Table I, we see that electrode $\mathbf{O 2}$ has a HTER of $0.12 \pm 0.04$, which is the minimum when compared to other electrodes.
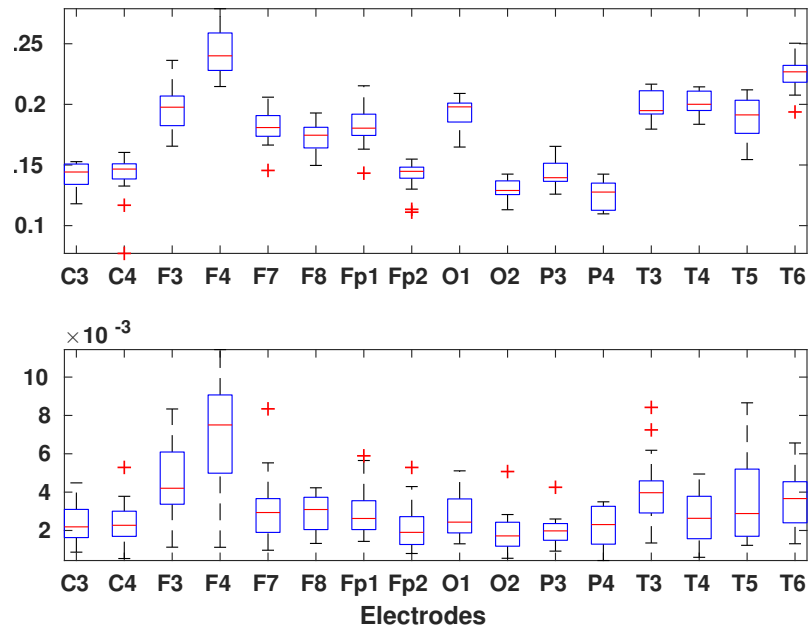

Fig. 3. Means (top) and standard deviations (bottom) of the HTER across 20 runs of 10 fold cross validation.

\section{Cross Subject Evaluation}

In the previous experiments, we were evaluating the performance at epoch level, where the training and test sets contained EEG signals from the same subjects. We suspect that this will have positively biased the performance of our classification model. Therefore, we created subject-level 10fold cross validation, as shown in Table II, to allow training using one set of subjects and testing on a separate set of subjects. The partitioning was carried out in such a way that at least one subject with $\mathrm{AD}$ is always present in the test set.

The results from Table III show an increase in the HTER
TABLE II. Number OF SUbJECTS CONSIDERED IN THE TRAINING AND TEST SETS ACROSS 10 FOLDS.

\begin{tabular}{|l|l|l|l|l|l|l|l|l|l|l|}
\hline Train size & 20 & 19 & 19 & 20 & 20 & 20 & 20 & 20 & 20 & 20 \\
\hline Test size & 2 & 3 & 3 & 2 & 2 & 2 & 2 & 2 & 2 & 2 \\
\hline
\end{tabular}

across all electrodes for different LBP segment sizes. This validates our hypothesis that the classification model reported earlier was positively biased by epoch-level cross validation; and the subject-level cross validation can mitigate this bias.

TABLE III. LBP PARAMETER TUNING USING CROSS SUBJECT EVALUATION.

\begin{tabular}{|c|c|c|c|c|}
\hline Electrode & \multicolumn{4}{|c|}{ LBP segment size (size of feature vector) } \\
\hline & $\mathbf{3}(\mathbf{4})$ & $\mathbf{5}(\mathbf{1 6})$ & $\mathbf{7}(\mathbf{6 4 )}$ & $\mathbf{9} \mathbf{( 2 5 6 )}$ \\
\hline C3 & $0.29 \pm 0.16$ & $0.29 \pm 0.18$ & $0.27 \pm 0.17$ & $0.50 \pm 0.00$ \\
\hline C4 & $0.34 \pm 0.15$ & $0.32 \pm 0.16$ & $0.36 \pm 0.14$ & $0.50 \pm 0.00$ \\
\hline F3 & $0.34 \pm 0.14$ & $0.33 \pm 0.15$ & $0.30 \pm 0.17$ & $0.50 \pm 0.00$ \\
\hline F4 & $0.41 \pm 0.10$ & $0.41 \pm 0.12$ & $0.35 \pm 0.16$ & $0.50 \pm 0.00$ \\
\hline F7 & $0.28 \pm 0.16$ & $0.35 \pm 0.15$ & $0.30 \pm 0.17$ & $0.50 \pm 0.00$ \\
\hline F8 & $0.32 \pm 0.15$ & $0.37 \pm 0.13$ & $0.38 \pm 0.14$ & $0.50 \pm 0.00$ \\
\hline Fp1 & $0.37 \pm 0.14$ & $0.37 \pm 0.15$ & $0.30 \pm 0.18$ & $0.50 \pm 0.00$ \\
\hline Fp2 & $0.36 \pm 0.15$ & $0.35 \pm 0.15$ & $0.32 \pm 0.18$ & $0.50 \pm 0.00$ \\
\hline O1 & $0.34 \pm 0.17$ & $0.34 \pm 0.15$ & $0.29 \pm 0.15$ & $0.50 \pm 0.00$ \\
\hline O2 & $0.38 \pm 0.13$ & $\mathbf{0 . 2 7} \pm \mathbf{0 . 1 5}$ & $0.31 \pm 0.18$ & $0.50 \pm 0.00$ \\
\hline P3 & $0.39 \pm 0.15$ & $0.40 \pm 0.12$ & $0.31 \pm 0.15$ & $0.50 \pm 0.00$ \\
\hline P4 & $0.33 \pm 0.16$ & $0.31 \pm 0.16$ & $0.30 \pm 0.18$ & $0.50 \pm 0.00$ \\
\hline T3 & $0.39 \pm 0.14$ & $0.40 \pm 0.11$ & $0.32 \pm 0.17$ & $0.50 \pm 0.00$ \\
\hline T4 & $0.38 \pm 0.13$ & $0.31 \pm 0.17$ & $0.40 \pm 0.14$ & $0.50 \pm 0.00$ \\
\hline T5 & $0.41 \pm 0.15$ & $0.39 \pm 0.14$ & $0.35 \pm 0.16$ & $0.50 \pm 0.00$ \\
\hline T6 & $0.41 \pm 0.11$ & $0.44 \pm 0.11$ & $0.30 \pm 0.17$ & $0.48 \pm 0.07$ \\
\hline
\end{tabular}

Although we see an increase in the HTER across all the electrodes, the results in Figure 3 are consistent with the results shown in Figure 4. Electrode $\mathbf{O 2}$ also still proved to be the best at discriminating between the $\mathrm{AD}$ and control patients.
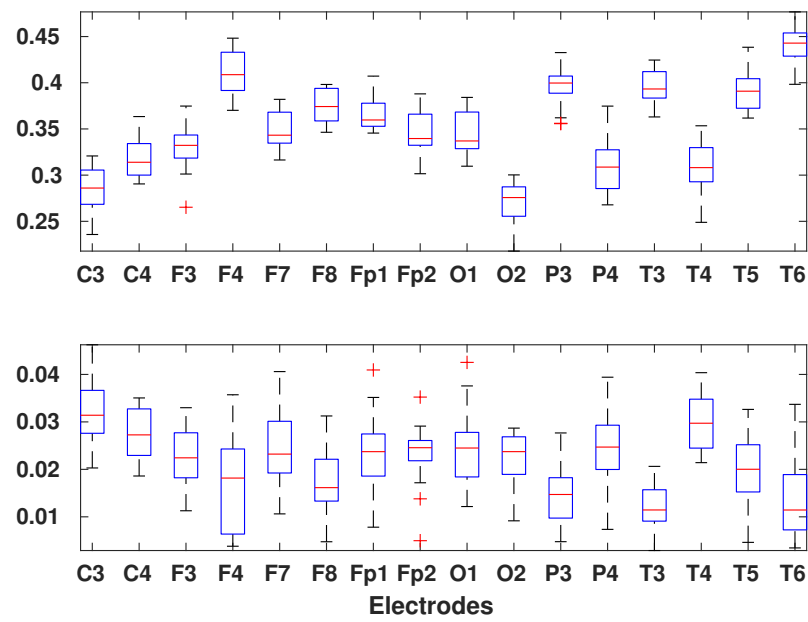

Fig. 4. Means (top) and standard deviations (bottom) of the HTER across 20 runs of 10 fold cross validation using cross subject evaluation.

\section{Discussions AND CONCLUSIONS}

In this study, we presented a method using 1D-LBPs for effectively discriminating between $\mathrm{AD}$ and control patients based on their EEG signals. Our preliminary observation showed differences between the groups, with $\mathrm{AD}$ patients' 1D-LBP histograms having greater levels of intensity when compared to the controls. Our implementation included 20 repetitions of a 10-fold cross validation across all 16 electrodes. Furthermore, we found that 1D-LBP with a segment 
size of 5 gave the best performance (i.e., with the minimum HTER value) when compared to other segment sizes. Please note that, we have reported only the performance of single electrodes to demonstrate the viability of the 1D-LBP method, as a preliminary study. In practice, however, all electrodes must be used jointly through multivariate methods, including feature selection and feature extraction (projection into lower dimensional space), which will be constituted in our future study.

Our results showed that mean HTER varies substantively between electrodes both in the epoch- and subject-level experiments. In the case of the epoch-level experiments, electrodes covering the frontal and temporal lobes were consistently less useful in discriminating between $\mathrm{AD}$ and healthy subjects. However, this pattern was not observed in the subject-level experiments, with no discernible trend in performance across lobes or hemispheres. Despite this, electrodes C3, C4, O2 and P4 showed high discriminative power across both experiments. Previous research using this database and non-linear analysis methods has highlighted AD changes mostly at electrodes in the parietal $(\mathrm{P} 3, \mathrm{P} 4)$ and occipital $(\mathrm{O} 1, \mathrm{O} 2)$ regions, but rarely in the central region $(\mathrm{C} 3, \mathrm{C} 4)$ [26]. Therefore, our preliminary results suggest that the method presented in this paper could provide additional information with diagnostic potential and complement what is already known about the EEG in AD. Nevertheless, further studies with a much greater sample size are required to test the possible clinical usefulness of this methodology.

\section{ACKNOWLEDGEMENT}

The authors would like to acknowledge Dr. Simon Bull for his contribution in preparation of this paper. ST and NP benefited from the Medical Research Council (MRC)-funded project 'Modelling the Progression of Chronic Kidney Disease' (R/M023281/1) www.modellingCKD.org.

\section{REFERENCES}

[1] M. Graeber, S. Kösel, R. Egensperger, R. Banati, U. Müller, K. Bise, P. Hoff, H. Möller, K. Fujisawa, and P. Mehraein, "Rediscovery of the case described by Alois Alzheimer in 1911: historical, histological and molecular genetic analysis," Neurogenetics, vol. 1, no. 1, pp. 73-80, 1997.

[2] G. M. McKhann, D. S. Knopman, H. Chertkow, B. T. Hyman, C. R. Jack, C. H. Kawas, W. E. Klunk, W. J. Koroshetz, J. J. Manly, R. Mayeux et al., "The diagnosis of dementia due to Alzheimers disease: Recommendations from the National Institute on Aging-Alzheimers association workgroups on diagnostic guidelines for Alzheimer's disease," Alzheimer's \& dementia, vol. 7, no. 3, pp. 263269, 2011.

[3] D. S. Knopman, S. T. DeKosky, J. Cummings, H. Chui, J. Corey-Bloom, N. Relkin, G. Small, B. Miller, and J. Stevens, "Practice parameter: Diagnosis of dementia (an evidence-based review) report of the quality standards subcommittee of the American Academy of Neurology," Neurology, vol. 56, no. 9, pp. 1143-1153, 2001.

[4] B. Reisberg, R. Doody, A. Stöffler, F. Schmitt, S. Ferris, and H. J. Möbius, "Memantine in moderate-to-severe Alzheimer's disease," New England Journal of Medicine, vol. 348, no. 14, pp. 1333-1341, 2003.

[5] L. A. Coben, W. L. Danziger, and L. Berg, "Frequency analysis of the resting awake EEG in mild senile dementia of Alzheimer type," Electroencephalography and clinical neurophysiology, vol. 55, no. 4, pp. 372-380, 1983.

[6] F. Miraglia, F. Vecchio, and P. M. Rossini, "Searching for signs of aging and dementia in EEG through network analysis," Behavioural brain research, vol. 317, pp. 292-300, 2017.
[7] R. Gandelman-Marton, S. Aichenbaum, E. Dobronevsky, M. Khaigrekht, and J. M. Rabey, "Quantitative EEG after brain stimulation and cognitive training in Alzheimer disease," Journal of Clinical Neurophysiology, vol. 34, no. 1, pp. 49-54, 2017.

[8] M. Chochoi, L. Tyvaert, P. Derambure, and W. Szurhaj, "Is long-term electroencephalogram more appropriate than standard electroencephalogram in the elderly?" Clinical Neurophysiology, vol. 128, no. 1, pp. 270-274, 2017.

[9] J. Jeong, "EEG dynamics in patients with Alzheimer's disease," Clinical neurophysiology, vol. 115, no. 7, pp. 1490-1505, 2004.

[10] D. Abásolo, R. Hornero, P. Espino, D. Alvarez, and J. Poza, “Entropy analysis of the EEG background activity in Alzheimer's disease patients," Physiological measurement, vol. 27, no. 3, p. 241, 2006.

[11] H. Azami, D. Abásolo, S. Simons, and J. Escudero, "Univariate and multivariate generalized multiscale entropy to characterise EEG signals in Alzheimers disease," Entropy, vol. 19, no. 1, p. 31, 2017.

[12] D. Abásolo, R. Hornero, P. Espino, J. Poza, C. I. Sánchez, and R. de la Rosa, "Analysis of regularity in the EEG background activity of Alzheimer's disease patients with Approximate Entropy," Clinical Neurophysiology, vol. 116, no. 8, pp. 1826-1834, 2005.

[13] D. Abásolo, R. Hornero, C. Gómez, M. García, and M. López, "Analysis of EEG background activity in Alzheimer's disease patients with Lempel-Ziv complexity and central tendency measure," Medical engineering \& physics, vol. 28, no. 4, pp. 315-322, 2006.

[14] C. J. Stam, "Nonlinear dynamical analysis of EEG and MEG: review of an emerging field," Clinical neurophysiology, vol. 116, no. 10, pp. 2266-2301, 2005.

[15] S. Simons, D. Abasolo, and J. Escudero, "Classification of Alzheimer's disease from quadratic sample entropy of electroencephalogram," Healthcare technology letters, vol. 2, no. 3, pp. 70-73, 2015.

[16] T. Ojala, M. Pietikäinen, and D. Harwood, "A comparative study of texture measures with classification based on featured distributions," Pattern recognition, vol. 29, no. 1, pp. 51-59, 1996.

[17] T. Ojala, M. Pietikäinen, and T. Mäenpää, "Gray scale and rotation invariant texture classification with local binary patterns," in European Conference on Computer Vision. Springer, 2000, pp. 404-420.

[18] N. Chatlani and J. J. Soraghan, "Local binary patterns for 1-D signal processing," in Signal Processing Conference, 2010 18th European. IEEE, 2010, pp. 95-99.

[19] Y. Kaya, M. Uyar, R. Tekin, and S. Yıldırım, "1D-local binary pattern based feature extraction for classification of epileptic EEG signals," Applied Mathematics and Computation, vol. 243, pp. 209-219, 2014.

[20] A. Şengür, Y. Guo, and Y. Akbulut, "Time-frequency texture descriptors of EEG signals for efficient detection of epileptic seizure," Brain Informatics, vol. 3, no. 2, pp. 101-108, 2016.

[21] L. Boubchir, S. Al-Maadeed, A. Bouridane, and A. A. Chérif, "Timefrequency image descriptors-based features for EEG epileptic seizure activities detection and classification," in Acoustics, Speech and Signal Processing (ICASSP), 2015 IEEE International Conference on. IEEE, 2015, pp. 867-871.

[22] S. Tirunagari, N. Poh, M. Bober, and D. Windridge, "Windowed $\mathrm{DMD}$ as a microtexture descriptor for finger vein counter-spoofing in biometrics," in Information Forensics and Security (WIFS), 2015 IEEE International Workshop on. IEEE, 2015, pp. 1-6.

[23] S. Tirunagari, N. Poh, D. Windridge, A. Iorliam, N. Suki, and A. T. Ho, "Detection of face spoofing using visual dynamics," IEEE transactions on information forensics and security, vol. 10, no. 4, pp. 762-777, 2015.

[24] Q. Zhu, N. Chatlani, and J. J. Soraghan, "1-D local binary patterns based VAD used in HMM-based improved speech recognition," in Signal Processing Conference (EUSIPCO), 2012 Proceedings of the 20th European. IEEE, 2012, pp. 1633-1637.

[25] S. Kouchaki, S. Tirunagari, A. Tapinos, and D. L. Robertson, "Local binary patterns as a feature descriptor in alignment-free visualisation of metagenomic data," in 2016 IEEE Symposium on Computational Intelligence and Data Mining (CIDM), 2016.

[26] D. Abásolo, J. Escudero, R. Hornero, C. Gómez, and P. Espino, "Approximate entropy and auto mutual information analysis of the electroencephalogram in alzheimers disease patients," Medical and Biological Engineering and Computing, vol. 46, no. 10, pp. 1019-1028, 2008 . 